\title{
Self-adaptive Improvement of Firefly Algorithm for Solving Multimodal Function
}

\author{
Xiaojie Liu \\ College of Computer Science and Technology, Jilin University, Changchun, Jilin, 130012, China. \\ 1553316673@qq.com
}

Keywords: Firefly Algorithm; Self-adaptive; Change Step; Multimodal Function.

Abstract. According to the problem that firefly algorithm converges slowly, easily falls into local optimum value and cannot acquire solutions exactly for solving the multimodal function, a self-adaptive firefly algorithm was proposed through analysis and research. In this new algorithm, parameter $\gamma$ and parameter $\alpha$ are self-adaptive, rather than constant. The experimental results of four standard test functions show that this new method is superior to firefly algorithm in convergence and computational precision.

\section{Introduction}

All Swarm intelligence optimization is a bionic algorithm, which can simulate evolution. It was abstracted from the law of population cooperation. It provides a new way to solve the complicated optimization problems by taking advantage of the group and simulating the process of animal' $\mathrm{s}$ foraging or information interaction between individuals. Due to the characteristics of simple operation, high efficiency and strong stability, swarm intelligence optimization algorithm has been applied to many fields, such as neural network training [1], image processing [2], data mining [3], and so on. Particle swarm optimization and ant colony optimization [4] are more commonly used swarm intelligence algorithm.

Firefly algorithm (FA) is an optimization algorithm based on population search, which is proposed by Yang [5], a Cambridge scholar. The idea comes from that fireflies glow in the nature, and each firefly will follow others which are brighter than itself in the range of vision.

Although FA has been applied to many fields, there are some defects [6], such as slow convergence speed and low accuracy of solving. In this paper, the basic firefly algorithm is analyzed, and a self-adaptive firefly algorithm (SAFA) is proposed. In this new algorithm, parameter $\gamma \square$ (light absorption coefficient) and parameter $\alpha \square$ (step factor) are improved. At last, the new algorithm' $\mathrm{s}$ performance is tested through four standard multimodal functions.

\section{Firefly Algorithm}

Bionic Principle. In nature, many kinds of fireflies can emit rhythmical and short fluorescence. The biological significance of this fluorescence may be attracting the opposite sex, helping to hunt, or as a warning signal. In FA, the biological significance of fluorescence is abandoned, and making the following assumptions [7].

1. All fireflies are regarded as the same gender, and they will be attracted to follow brighter fireflies.

2. For any two fireflies, the firefly whose brightness is weaker will move to the other. The brightness is inversely proportional to the distance.

3. If the firefly is the brightest one in the range of vision, it will move randomly.

In FA, there are two important factors. One is the brightness, and the other is attractiveness. Attraction between fireflies depends on the two factors. The brightness depends on the target, and the target depends on the location of the firefly. The stronger brightness represents the better target. Fireflies move to stronger brightness, which means the target becomes better and better. The attractiveness is related to the brightness. The brightness is stronger, and the attractiveness is stronger. 
The both factors are inversely proportional to the distance. This simulates the attenuation characteristic in the process of the fluorescence transmission.

The bionic principle of FA is that: 1) points in the search space are simulated as firefly individuals, 2) the function value of the point is simulated as the brightness of firefly, 3) the process of search and optimization is simulated as the process of attraction and movement between fireflies.

Mathematical Description. There are two factors in FA, which are the brightness and the attractiveness. The brightness determines the moving direction of fireflies. And the attractiveness determines the moving distance. As iteration going, fireflies whose brightness is weaker will close to the others whose brightness is stronger constantly. Finally, most fireflies will be gathered around the brightest one. The brightness of the brightest firefly is the optimal solution of question. The following is mathematical description [8].

Step 1 Initialization.

In FA, $n$ represents the number of fireflies in the search space, $\beta_{0}$ represents the biggest attractiveness, $\gamma$ represents light absorption coefficient, $\alpha$ represents step factor, and MaxGeneration represents the number of iterations. They will be initialized at the beginning of the experiment. $\mathrm{I}_{0}$ represents the biggest brightness of each of fireflies, and it will be initialized the function value $\mathrm{f}(\mathrm{x})$.

Step 2 Calculate the distance between the firefly $i$ and the firefly $j$.

$$
R_{i j}=\left\|X_{i}-X_{j}\right\|
$$

In the formula, $X_{i}$ represents the location of the firefly $i, X_{j}$ represents the location of the firefly $j$, and $\mathrm{R}_{\mathrm{ij}}$ is the distance.

Step 3 Calculate the attractiveness between the firefly $i$ and the firefly $j$.

$$
\beta=\beta_{0} \times e^{-\gamma R i j^{2}} \cdot \beta_{0} \in[0,1], \gamma \in[0.01,100]
$$

Step 4 Update the location of firefly

If the brightness of the firefly $i$ is weaker than the brightness of the firefly $j, i$ will be close to $j$. On the contrary, $\mathrm{j}$ will be close to $\mathrm{i}$. Take the first case for example. Then the location of $\mathrm{i}$ will change as follows:

$$
X_{i}(t+1)=X_{i}(t)+\beta \times\left(X_{j}(t)-X_{i}(t)\right)+\alpha \times(\text { rand }-0.5) . \alpha \in[0,1]
$$

In the formula, the first part represents the location of the firefly $i$ when number of iterations is $t$. The second part reflects the information sharing between fireflies. Random step is set in the third part to avoid falling into a local optimum. $\alpha$ represents step factor.

Step 5 The brightest firefly flies randomly.

In one iteration, the brightest firefly will fly randomly as follows.

$$
X_{b}(t+1)=X_{b}(t)+\alpha \times(\text { rand }-0.5) . \alpha \in[0,1]
$$

In the formula, $X_{b}(t)$ is global optimum location when number of iterations is $t$. The firefly which at the global optimum location will fly randomly. $X_{b}(t+1)$ represents the location of the firefly next iteration.

Step 6 If the given iteration number or precision is reached, the algorithm will stops; otherwise, $\mathrm{t}=\mathrm{t}+1$, go to step 2 . 


\section{Algorithm Analysis and Improvement}

In this paper, light absorption coefficient and step factor are also improved, and a new algorithm is proposed, named self-adaptive firefly algorithm (SAFA).

Improve Parameter $\gamma$. For the basic firefly algorithm, parameter $\gamma$ (light absorption coefficient) shows that the brightness of fluorescence varies with transmission distance and media. The value of parameter $\gamma$ is in the interval $[0.01,100]$, it is set a fixed value usually. It can be seen from the above formula (2) that when the distance between the firefly $i$ and $j$ is very long, $e^{-R i j 2}$ will be close to zero, and $\beta$ will be close to zero too. Then the formula (3) will change to the formula (5).

$$
X_{i}(t+1) \approx X_{i}(t)+\alpha \times(\text { rand }-0.5) \cdot \alpha \in[0,1]
$$

It can be seen that when the distance between the firefly $\mathrm{i}$ and $\mathrm{j}$ is very long, the attraction between them is negligible. At this time, the firefly i will fly around itself randomly. It will lead to FA' s search results become worse and the convergence rate become slower.

In order to solve the problem, parameter $\gamma$ is improved. It is no longer a fixed value, but can adaptively adjust according to the distance. The improved parameter $\gamma$ is as follows.

$$
\gamma=\frac{\gamma_{0}}{\left\|R_{b}-R_{w}\right\| \times\left\|R_{b}-R_{i}\right\|} \cdot \gamma_{0} \in[0.04,4]
$$

In this formula, $R_{b}$ is the optimal location, $R_{w}$ is the worst location, $R_{i}$ is the current location of the firefly $i,\left\|R_{b}-R_{w}\right\|$ is the distance between the optimal location and the worst location, $\left\|R_{b}-R_{i}\right\|$ is the distance between the optimal location and the current location. Because $\mathrm{e}^{-4} \approx 0.0183$ and $\mathrm{e}^{-0.04} \approx$ 0.9608 , it is helpful to maintain a good communication among fireflies when $\gamma_{0}$ is in the interval $[0.04,4]$.

Combining formula (2) and (6), a very important point can be known. If the distance between groups is long, parameter $\gamma$ will be small to ensure the attractiveness. When the distance becomes shorter, the value of parameter $\gamma$ will become larger to enhance the ability of global search. Parameter $\gamma$ is self-adaptive through this improvement, and it can adjust according to the distance.

Improve Parameter $\boldsymbol{\alpha}$. For the basic firefly algorithm, parameter $\boldsymbol{\alpha}$ (step factor) is usually set a fixed value between 0 and 1 . If the value is large, the ability of global search will be strong, but it's possible to skip the global optimum because the step is too large. This will reduce the search accuracy. If the value is small, the ability of local search will be strong, but it will reduce the convergence speed. Obviously, the use of a fixed step does not meet the needs of the actual calculation process. In order to balance the relationship between global search and local search, parameter $\alpha$ is improved by using self-adaptive step.

The value of can adjust according to the number of iterations. At the beginning of the iteration, the value of parameter $\alpha$ is larger to keep strong ability of global search. With the iteration going on, the value becomes smaller to enhance the ability of local search. The improved parameter $\alpha$ is as follows.

$$
\alpha=e^{\frac{-4 \times t}{\text { MaxGeneration }}}
$$

In this formula, $\mathrm{t}$ represents the current number of iterations, MaxGeneration represents the maximum number of iterations. It is obvious that step factor decreases with the iteration going on. In the iterative process, the global searching ability is gradually weakened, and the local searching ability is gradually strengthened, which is helpful to find the global optimal solution. 


\section{Simulation Results and Analysis}

In order to test the performance of SAFA, four standard test functions are selected to do the simulation experiment. Then, compare SAFA with FA about experimental results.

Experiment Operation Platform. The operating environment of the experiment: processor is Intel Core i3-5010U, RAM is 8GB, operating system is Windows7, and Simulation environment is Matlab R2014a.

Experiment Parameters Setting. The number of fireflies $n=80$, light absorption coefficient $\gamma=1.0$, the biggest attractiveness $\beta_{0}=1.0$, step factor $\alpha=0.2$, the maximum number of iterations MaxGeneration $=100$. In SAFA, $\gamma_{0}=0.5$.

Standard Test Functions. In the experiment, four standard functions [9] are selected to test, in order to verify the performance of SAFA in solving the global optimum of multimodal function.

1) De Jong Function F2:

$$
f_{1}(x)=100\left(x_{1}^{2}-x_{2}\right)^{2}+\left(1-x_{1}\right)^{2}, x_{1,2} \in[-2.048,2.048]
$$

The global minimum is 0 .

2) Becker and Lago:

$$
f_{2}(x)=\left(\left|x_{1}\right|-5\right)^{2}+\left(\left|x_{2}\right|+5\right)^{2}, x_{1,2} \in[-10,10]
$$

The global minimum is 25 .

3) RA-Rastrigin $(n=2)$ :

$$
f_{3}(x)=x_{1}^{2}+x_{2}^{2}-\cos 18 x_{1}-\cos 18 x_{2}, x_{1,2} \in[-1,1]
$$

The global minimum is -2 .

4) Schaffer Function F6:

$$
f_{4}(x)=0.5+\frac{\sin ^{2} \sqrt{x_{1}^{2}+x_{2}^{2}}-0.5}{\left[1.0+0.001 \times\left(x_{1}^{2}+x_{2}^{2}\right)\right]^{2}}, x_{1,2} \in[-10,10]
$$

The global minimum is 0 .

Experiment Results. The best value, the worst value, the mean value and the standard deviation are obtained by testing $\mathrm{fl}(\mathrm{x}) \sim \mathrm{f} 4(\mathrm{x})$ independently for 20 times. The experimental data is shown in the table 1. It is clearly seen the comparison between SAFA and FA. In these data, the mean value reflects the accuracy of the solution, and the standard deviation reflects the stability of the algorithm. Since these test functions have a global minimum, the closer to the minimum means the higher solution accuracy. The smaller the standard deviation is, the more stable the algorithm is. 
TABLE 1. Performance comparison between SAFA and FA.

\begin{tabular}{cccccc}
\hline $\begin{array}{c}\text { Test } \\
\text { Function }\end{array}$ & Algorithm & $\begin{array}{c}\text { The Best } \\
\text { Value }\end{array}$ & $\begin{array}{c}\text { The Worst } \\
\text { Value }\end{array}$ & $\begin{array}{c}\text { The Mean } \\
\text { Value }\end{array}$ & $\begin{array}{c}\text { The } \\
\text { Standard } \\
\text { Deviation }\end{array}$ \\
\hline \multirow{2}{*}{$\mathrm{F}_{1}$} & SAFA & $6.74 \mathrm{e}-06$ & $8.04 \mathrm{e}-03$ & $1.39 \mathrm{e}-03$ & $2.98 \mathrm{e}-03$ \\
& FA & $2.20 \mathrm{e}-03$ & 0.663 & 0.314 & 0.249 \\
$\mathrm{~F}_{2}$ & SAFA & 25.00221 & 25.03011 & 25.01079 & 0.01125 \\
& FA & 25.01262 & 25.78811 & 25.20087 & 0.329518 \\
$\mathrm{~F}_{3}$ & SAFA & -1.99979 & -1.87730 & -1.95888 & 0.06291 \\
& FA & -1.86410 & -1.01588 & -1.64475 & 0.31795 \\
$\mathrm{~F}_{4}$ & SAFA & $9.71591 \mathrm{e}-02$ & $9.71743 \mathrm{e}-02$ & $9.71624 \mathrm{e}-02$ & $5.96159 \mathrm{e}-07$ \\
& FA & $9.71924 \mathrm{e}-02$ & $9.85193 \mathrm{e}-02$ & $9.76400 \mathrm{e}-02$ & $4.97114 \mathrm{e}-05$ \\
\hline
\end{tabular}

Fig. 1 Fig. 4 are four figures, which show the convergence of SAFA and FA.

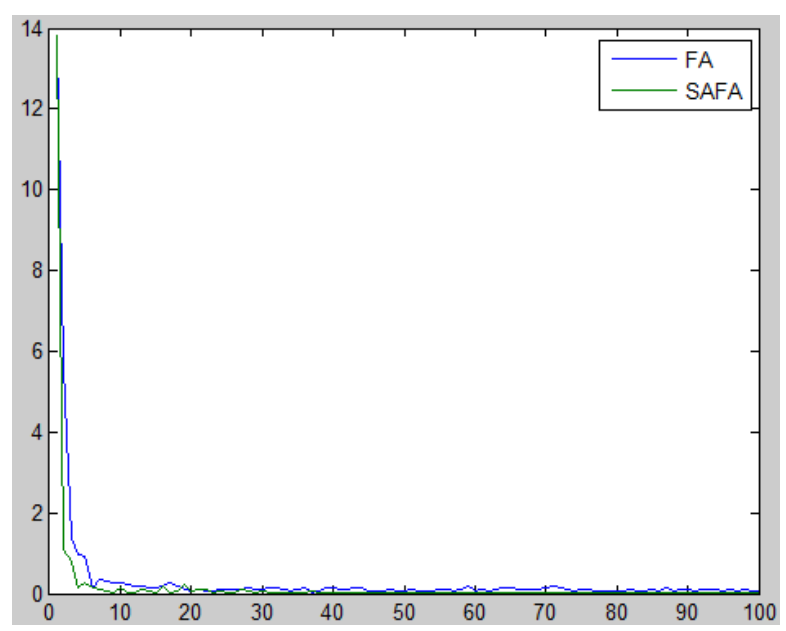

Fig. 1 Convergence curve of $f_{1}$

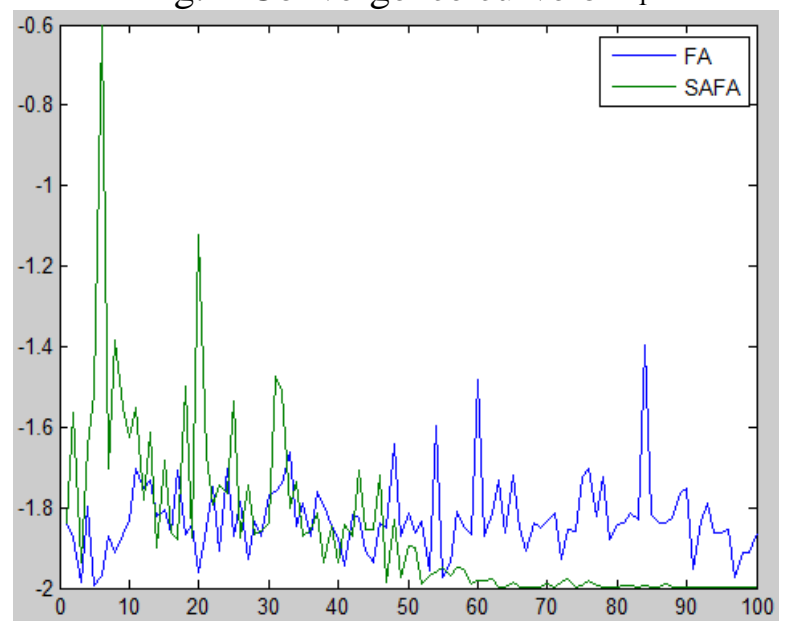

Fig. 3 Convergence curve of $f_{3}$

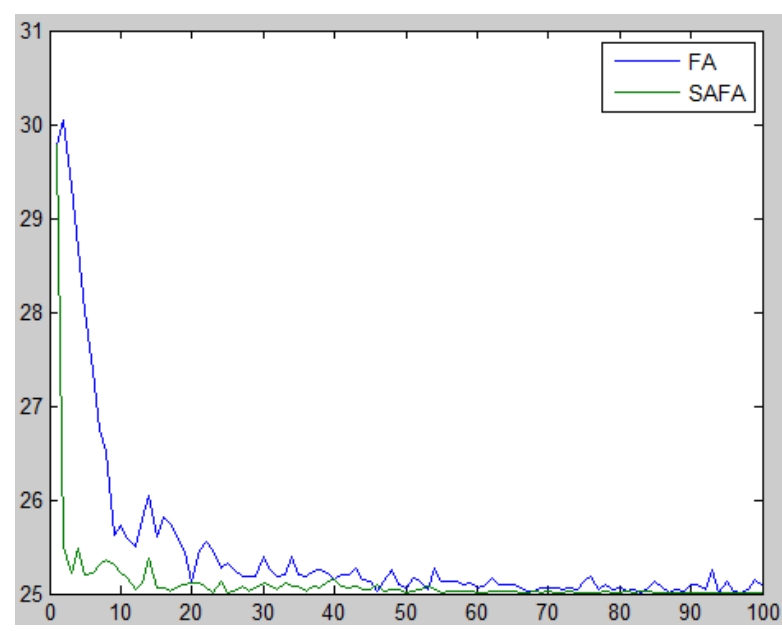

Fig. 2 Convergence curve of $f_{2}$

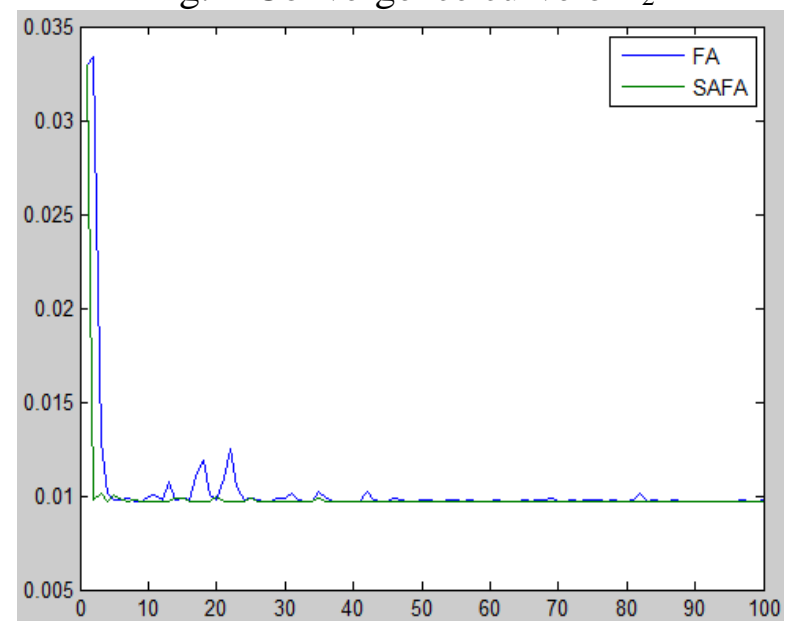

Fig. 4 Convergence curve of $f_{4}$

Results Analysis. From table 1, it can be seen that compared with FA, the solution value of SAFA is closer to the standard value, so SAFA is superior to FA in solving precision. The standard deviation of SAFA is lower than that of FA, so the stability of SAFA is stronger.In Fig.1 Fig.4, the curve shows that functions converge faster by using SAFA. So convergence of SAFA is stronger too. 


\section{Conclusions}

A self-adaptive firefly algorithm (SAFA) is proposed to solve the problem that the convergence speed is slow and the precision is not high enough when multimodal function is solved by firefly algorithm (FA). The new algorithm use self-adaptive light absorption coefficient which can adjust itself according to the distance to keep the good communication between groups and improve the ability of group cooperation. The step factor can adjust according to the number of iterations. It decreases with the increase of iteration number. At the beginning of the iteration, step factor is larger to keep higher ability of global search. In the latter part if the iteration, step factor is smaller to enhance the ability of local search. Simulation results show that SAFA is superior to FA in accuracy and convergence speed.

\section{Acknowledgments}

As a graduate student of Jilin University, I thank my Alma mater for providing me with a good educational environment. And many people have made invaluable contributions, both directly and indirectly to my research. I would like to express my warmest gratitude to professor Wang and my classmates. They gave me great encouragement and help. Finally, I greatly appreciate my parents. They always love me and behind me. My heart swells with gratitude to all the people who helped me. I wish to say " Thanks" !

\section{References}

[1] Horng M H, Lee Y X, Lee M C, et al. Firefly Meta-Heuristic Algorithm for Training the Radial Basis Function Network for Data Classification and Disease Diagnosis[M]// Theory and New Applications of Swarm Intelligence. 2012.

[2] Horng M H, Liou R J. Multilevel minimum cross entropy threshold selection based on the firefly algorithm[J]. Expert Systems with Applications, 2011, 38(12):14805-14811.

[3] Senthilnath J, Omkar S N, Mani V. Clustering using firefly algorithm: Performance study[J]. Swarm \& Evolutionary Computation, 2011, 1(3):164-171.

[4] Xuemei Li, Suqin Zhang. Overview of some optimization algorithm based on bionic theory[J]. Application Research of Computers, 2009, 26(6):2032-2034.

[5] Yang X S. Nature-Inspired Metaheuristic Algorithms[M]. Luniver Press, 2010.

[6] Changping Liu, Chunming Ye. Firefly Algorithm with Chaotic Search Strategy[J]. Journal of System \& Management, 2013, 22(4):538-543.

[7] Yanzhui Ma. Improved and Applied Research of Firefly Algorithm[D]. Guangxi University for Nationalities, 2014.

[8] Changping Liu, Chunming Ye. Novel bioinspired swarm intelligence optimization algorithm: firefly algorithm[J]. Application Research of Computers, 2011, 28(9):3295-3297.

[9] Shuhao Yu. Research on Firefly Algorithm and Its Application[D]. Hefei University of Technology, 2015. 